\title{
Prévention et crédibilité des politiciens: un champ de mines
}

\author{
Le rejet de l'initiative «Protection contre le tabagisme passif» par le Conseil natio- \\ nal, en décembre 2011, et au Conseil des Etats en mars 2012, revient à valider \\ a posteriori une loi fédérale déficiente. C'est incompréhensible.
}

Rainer M. Kaelin

* Les références se trouvent sous www.bullmed.ch $\rightarrow$ Numéro actuel ou $\rightarrow$ Archives $\rightarrow 2012 \rightarrow 23$.
La prévention en matière de santé est un thème politiquement explosif. Que la prévention du tabagisme soit influencée massivement par le lobby du tabac ne fait guère de doute. Cependant, que les parlementaires Suisses se laissent distraire de leur mandat au point de refuser l'initiative «Protection contre le tabagisme passif» laisse songeur: en effet, ce rejet par les Chambres constitue un applaudissement tardif à la loi fédérale d'octobre 2008.

\section{L'élaboration de la loi fédérale de 2008 sur la protection de la fumée passive}

En 2004, le député et professeur de médecine Felix Gutzwiller (PLR/ZH), déposa l'initiative «Protection de la population et de l'économie contre la fumée passive» avec pour objectif un lieu de travail sans fumée. La même année, le ministre de la santé et conseiller fédéral Pascal Couchepin signait la convention cadre pour le contrôle du tabagisme de l'OMS [1] ${ }^{*}$, qui stipule que «les parties signataires reconnaissent que les travaux scientifiques prouvent sans équivoque que la fumée passive cause la mort, la maladie et l'invalidité».

Une sous-commission de la commission de la santé du Conseil national (CS-N) élabora un projet de loi, qui fut présenté en juin 2007 par Pierre Triponez (PLR/BE), président de la CS-N: «Cette proposition de loi amène un changement de paradigme. Le lieu de travail et les espaces fermés accessibles au public devront par principe être libres de fumée. Cette règle concerne aussi les locaux de la gastronomie» [2]. La CS-N d'alors opta avec 16 voix contre 3 pour un texte de loi spécifique (et non pour une modification de la Loi sur le travail).

Puis, par étapes, cet estimable projet fut démantelé. Le 4 octobre 2007, suivant la minorité de la CS-N, le Conseil national adopta un projet prévoyant que des établissement publics auraient le droit de se déclarer locaux fumeurs, si une séparation entre zone fumeurs et zone non-fumeurs n'était pas possible ou pas exigible [3]. Bien que la commission de la santé du Conseil des États se soit déclarée «en principe pour une protection contre la fumée passive», [4] elle chercha des solutions visant à permettre aux exploitants de faire travailler des em- ployé/es dans de tels espaces fumeurs. Ainsi, Bruno Frick (PDC/SZ, Conseil des Etats) proposa que le personnel pourrait travailler dans les fumoirs et les espaces fumeurs, pour autant qu'il y consente en signant son contrat de travail.

L'actuelle loi fédérale garde donc seulement le principe d'un espace public fermé sans fumée, mais avec de nombreuses exceptions: si l'établissement est plus petit que $80 \mathrm{~m}^{2}$, le restaurateur a le droit de le déclarer local fumeur; les fumoirs sont admis, et sont en principe sans service, sauf si les employés consentent à se faire enfumer; la loi est en vigueur sur l' ensemble du territoire suisse, excepté dans les cantons avec loi plus contraignante.

Cette loi fut adoptée de justesse avec 89 voix contre 88 au Conseil national et au Conseil des Etats, avec la voix prépondérante du président Christoffel Brändli (UDC/GR), alors président de santésuisse. A vrai dire, il ne s'agit pas d'une loi fédérale, mais d'une loi alibi minimale (voir encadré, page suivante), puisqu'elle prévoit expressément que les cantons édictent des règles plus strictes.

\section{Débat et vote du conseil national (22 décembre 2011)}

Face à une loi à ce point vidée de sa substance, il était logique qu'une alliance d'organisations de santé et d'associations médicales, dont la FMH (www.sansfumée-oui.ch [6]) lançât une initiative populaire, intitulée «Protection contre le tabagisme passif», qui fut déposé en mai 2010. Le refus de cette initiative par le Conseil national par 118 voix contre 55 (et par le Conseil des Etats, qui refusa par 26 contre 15 voix un renvoi en commission), constitue un soutien tardif, mais massif à la loi fédérale. C'est d'autant plus étonnant qu'à l'origine, celle-ci avait été acceptée d'extrême justesse. Quels sont donc les arguments qui aujourd'hui, sont apparus tellement plus convaincants qu'il y a 4 ans?

- Mme Humbel Naef (PDC/AG) parle pour la commission de la santé, qui refuse l'initiative avec 14 voix contre 8 . Elle trouve "problématique» de changer la loi, tout en constatant que 15 cantons connaissaient des règles plus strictes déjà avant que l'actuelle loi fût adoptée. Néan- 
moins on reproche à l'initiative d'être «disproportionnée» et de venir «trop tôt».

- On dénigre les auteurs de l' initiative en les accusant «d'entêtement» ou d'être de «mauvaise foi» (Daniel Fessler, CE/AI et Sylvia Flückiger Bäni (UDC/AG). Cette représentante du peuple et du comité de l'USAM, rappelle «le grand rôle social du bistrot», ce qui est pour elle une raison suffisante pour que les employés se fassent enfumer.

- L'argumentation se drape d'accents pathétiques: «Je suis pour une interdiction de la fumée en lieu public. Mais puisque, pour la plus grande majorité de nous, la santé est un bien très important, les décisions (la concernant) doivent être prises par chacun. Dans une communauté qui considère ses membres comme des mineurs (sic!), le législateur ne peut pas et ne doit pas se charger de la responsabilité des décisions à prendre» (Roland Büchel, UDC/SG). Confus, l'argumentaire de Pierre Rusconi (UDC/TI): le Tessin a donné le bon exemple, (et) a la chance d'avoir une bonne convivialité entre fumeurs et non-fumeurs. (...) Mais si l'on doit arriver à la perfection en continuant à réglementer n' importe quoi, notre perfection nous privera de toute liberté. Ce n'est peut-être pas encore le moment de faire un autre pas en avant. La bonne preuve donnée jusqu'à aujourd'hui est tout à fait suffisante. Je vous invite pourtant à ne pas accepter encore - je mets l'accent sur encore - cette initiative populaire.»

- On suggère un conflit avec la Constitution et la convention Cadre de l' OMS: «Il s'agit de garder une cohérence de notre ordre juridique. Notre lé-

\section{Et nous osons rappeler au législateur une exigence évidente: dans notre pays, tout le monde a le droit de respirer un air propre et libre de fumée.}

gislation actuelle a été édictée dans l'esprit du postulat de la convention cadre de l' OMS et dans le respect du principe de proportionnalité de notre Constitution fédérale; j'ai subi la tyrannie des fumeurs. Faut- il pour autant substituer une tyrannie à une autre?» (Mauro Poggia, sans parti/GE).

\section{Un combat déséquilibré}

Contre de tels sophismes, les 19 conseillers nationaux qui plaidaient pour l'adoption de l'initiative, étaient impuissants. Leurs interventions, à côté des arguments scientifiques connus, [7] étaient évidents: «Le contenu de l'initiative ne peut pas être si déraisonnable, car il est identique à la proposition de la commission de la santé de 2007. Si ce n'est pas l'ob-

\section{Critiques de la loi fédérale de 2008} lors de son élaboration [5]

- Le conseiller fédéral Pascal Couchepin défendait une solution, telle qu'elle est visée par l'initiative populaire «Protection contre le tabagisme passif»: «Les arguments économiques ... Honnêtement, ne sont pas sérieux! (...) Personne ne peut contester que la fumée passive soit quelque chose de négatif (...) Je vous invite à voter en faveur d'une solution qui n'est pas liberticide, qui est tout simplement saine, et à vous rallier à la solution du Conseil fédéral.»

- Konrad Graber (PDC/LU): «A quoi bon d'adopter une loi, qui aura comme conséquence que les cantons s'activent pour légiférer? Si l'on n'arrive pas à une solution (uniforme) pour toute la Suisse, on peut en effet se demander, si c'est utile d'édicter une loi.»

- Rolf Büttiker (PLR/SO): «Imaginez, de propager et d'instaurer dans ce pays 26 solutions différentes! (...) Ceci serait problématique du point de vue de la politique de l'Etat. C'est pourquoi, même en libéral, je voterai de manière cohérente la solution stricte, telle que le Conseil fédéral la préconise.»

- Urs Schwaller (PDC/FR/Conseil des Etats): «Dans la commission, l'enthousiasme pour la nouvelle loi était limité (...) Le résultat de 4 voix contre 3, avec 3 abstentions montre que nous avons déjà accompli (et) avec plus de conviction (...) du meilleur travail législatif.»

- Felix Gutzwiller (PLR/ZH): «La protection des travailleurs se doit d'être uniforme. II n'existe pas d'agent nocif, pour lequel on créerait deux types de postes de travail, où l'on dirait: s'il vous plaît cher(e)s employées et employés, vous êtes libres d'occuper un poste de travail avec exposition nocive ou sans exposition nocive.» Et: «Le secteur (de la gastronomie) d'emblée a combattu une solution du droit du travail, toujours avec l'argument principal, qu'on ne voulait pas d'inégalités. II y a des problèmes de délimitations: il y a des entreprises en gastronomie qui produisent des produits alimentaires, il y a des établissements dans des centres commerciaux qui sont libres de fumée, on ne pourrait donc pas y fumer, alors qu'à côté cela serait autorisé. Voyons, ceci n'est pas une législation sérieuse!»

jectif de toute entreprise de protéger ses employées et employés de la fumée passive..., des tels risques de santé sont à éliminer en première ligne» (Maya Ingold, CE/ZH).

«Cette manière de traiter la protection de la santé des travailleurs est une farce. Il n'est pas juste que sous la feuille de vigne de la responsabilité indivi- 


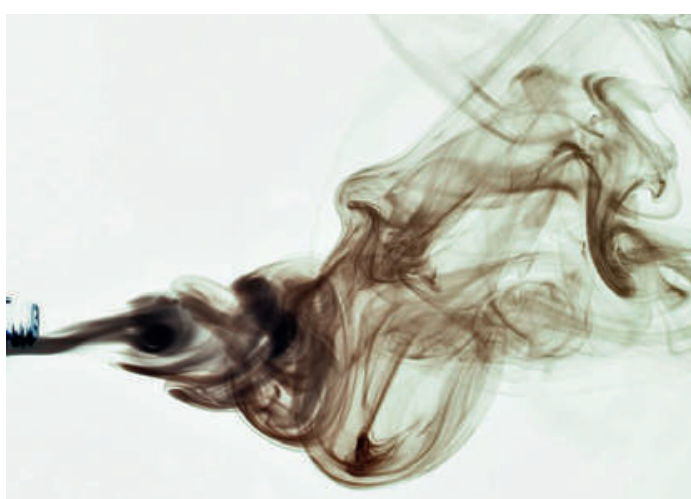

Tous les professionnels de la santé seront appelés à contribuer au succès de l'initiative «protection contre le tabagisme passif» lors de la votation en automne.

duelle, nous reportions la responsabilité de protéger la santé sur le lieu de travail sur les épaules des travailleuses et travailleurs. Cette évolution est rétrograde. Monsieur le conseiller fédéral, je m'étonne qu'un ministre de la santé se batte contre l'amélioration de la protection de la santé sur le lieu du travail! Pouvez vous exclure qu'un travailleur du secteur ne puisse encourir des sanctions, par exemple sous forme de non-paiements par l'assurance-chômage, s'il revendique le droit de refuser un travail dans un établissement fumeur, et qu'il perdrait son emploi pour cette raison?» (Max Chopart Acklin PS/AG).

\section{Didier Burkhalter: une position incompréhensible}

Le Conseil national ni la population Suisse, n'ont reçu une réponse valable du ministre de la santé en office en décembre 2011. Par rapport à son prédécesseur et collègue de parti Couchepin, qui aurait honte d'une loi pareille [8], la déclaration de M. Burkhalter est... diplomatique [9]. Il admet que la réglementation actuelle est incomplète, qu'elle est très en retrait par rapport à la solution défendue jadis par le Conseil fédéral, que la diminution des syndromes coronariens aigus est attribuable aux lois cantonales plus strictes (et non pas à la loi fédérale déjà dépassée lors de son adoption). Mais il recommande le refus de l'initiative!

\section{Lobbying et conflits d'intérêts}

- Les arguments avancés devant les Chambres ne justifient pas leur soutien massif à la loi fédérale, alors qu'elle fut adoptée du bout des lèvres en 2008. Que le lobbying ait joué un rôle déterminant semble évident: la situation actuelle profite en effet uniquement aux cigarettiers [10]. Dans les faits, leur message «la fumée passive n'est pas si grave que ça» se trouve renforcé par une loi fédérale déficiente. Des règles cantonales peuvent plus facilement être diluées en référence au modèle fédéral, et des tentatives pour esquiver la loi, par des clubs de fumeurs par exemple, obtiennent un semblant de caution juridique. La banalisation de la fumée, promue avec force auprès de la jeunesse $[11,12,13]$, est ainsi officialisée par un texte fédéral! Les efforts de l'industrie du tabac sont déjà couronnés de succès, comme le démontrent les chiffres récents: malgré une diminution du nombre total de fumeurs en Suisse, qui se situe actuellement à $27 \%$, le recul stagne depuis 2008. Et depuis 2010 le nombre des jeunes fumeurs réguliers (14-16 ans) a encore augmenté [14].

- L'analyse du vote des Chambres [15] montre que parmi les partis, l'UDC, le Parti bourgeois démocratique et les Verts libéraux ont voté sans abstention et de manière unanime contre une législation efficace pour la protection contre la fumée passive, suivis par les libéraux-radicaux et les démocrates chrétiens, alors que les socialistes et les Verts ont soutenu l'initiative. Comme déjà été indiqué $[17,18]$, des intérêts habilement masqués ont joué un rôle, comme le montre l'«Alliance pour une politique de prévention modérée» (www.aepm.ch [19]). Fondée 2008 par l'USAM, elle compte parmi ses membres Gastrosuisse, notoirement infiltrée par l'industrie de tabac [20], les associations de fabricants de cigares et de marchands de tabac; elle est soutenue par les partis politiques UDC, PDC et l'organisme «Swiss Cigarette», derrière lequel se cachent les trois multinationales du tabac installées en Suisse.

- Des interventions parlementaires au Conseil national du 22.12.2011 montrent que ces cercles exercent des pressions. «... la commission fédérale extraparlementaire du tabac, (...) a appelé à combler une fois pour toutes les lacunes dans la protection contre la fumée passive. Cette intrusion plus que problématique soulève des graves questions politiques. Il s'y ajoute que de telles commissions sont composées de manière parfaitement unilatérale. Tandis que les cercles du tabac n'y sont pas représentés, plusieurs membres de la commission sont de la ligue pulmonaire.» (Flückiger Bäni, UDC/AG). Et encore: «Comment se fait-il que la commission fédérale du tabac, payée et supervisée par la Confédération, donne son opinion et son avis après que le Conseil fédéral s'est déclaré, et prenne ainsi clairement position contre le Conseil fédéral?» (Roland Borer $\mathrm{UDC} / \mathrm{SO}$ ).

Le démontage systématique du «changement de paradigme» proposé en 2007, et le refus de l'initiative «Protection contre le tabagisme passif» par le Conseil fédéral et le parlement, nuisent à la crédibilité des autorités fédérales. Elles devraient faire prévaloir les intérêts de santé de la population, avant ceux de l'industrie tabac. Ceci n'est pas une question de politique politicienne de la part des partis, mais d'hon- 
nêteté intellectuelle. Nous sommes très inquiets de voir des parlementaires se laisser instrumentaliser pour critiquer la commission fédérale, précisément parce que celle-ci s'exprime sans être gênée par des conflits d'intérêts. Et nous osons rappeler au législateur une exigence évidente: dans notre pays, tout le monde a le droit de respirer un air propre et libre de fumée. Puisque les élu(e)s du peuple peinent à admettre cette évidence, ce sera bientôt le peuple qui le fera. Tous les professionnels de la santé seront appelés à contribuer au succès de l'initiative lors de la votation en automne.

Dr Erik Allgöwer, FMH Médecine interne, Yverdon-les-Bains

Dr. med. Jürg Barandun, Gründer und Leitender Partner Lungenzentrum Hirslanden Zürich

PD Dr. Jürg Barben, Präsident Lungenliga St. Gallen. Leitender Arzt Pneumologie/Allergologie. Ostschweizer Kinderspital, St. Gallen

Prof. Chris T. Bolliger, Professor of Medicine, Director Respiratory Research, Co-chairman Division of Pneumology, Faculty of Health Sciences, University of Stellenbosch, Cape Town, South Africa

Dr. med. Antonio Bonfiglio, FMH Allgemeinmedizin, Vorstandsmitglied SGAM, Langnau a.A.

Dr. med. Otto Brändli, Lungenarzt, Präsident Swiss Lung Fundation

Dr. med. Johannes Brühweiler, FMH Innere Medizin, Vorstandsmitglied Hausärzte Schweiz, Zürich

Prof. Dr. med. Martin Brutsche, Chefarzt Pneumologie und Zentrum Schlafmedizin, St. Gallen

Prof. em, Jean-Claude Chevrolet, ancien médecin chef des soins intensifs des HUG, Genève

Dr. med. Bernard Egger, Pneumologie FMH, médecin chef, Hôpital de Rolle/VD

Dr Nicolas Eisenhoffer, Pédiatrie FMH, Genolier

Dr. med. Margot Enz Kuhn, FMH Allgemeinmedizin, Vorstandsmitglied SGAM, Baden

Dr. med. Ingo Fengels, Pneumologie FMH, Vizepräsident und Ligaarzt, Lungenliga Luzern-Zug,

Vorstandsmitglied Krebsliga Zentralschweiz

Prof. Dr Jean-William Fitting, Médecin chef, Service de Pneumologie, CHUV, Lausanne

Dr Jean-Georges Frey, Pneumologie FMH, Médecin Sous-directeur, Centre valaisan de Pneumologie Montana

PD Dr. med. Christophe von Garnier, Leitender Arzt, Universitäts-Kinderklinik für Pneumologie, Inselspital Bern

Dr Stephen Garronne, FMH Pneumologie et Médecine interne, Monthey/VS

Prof. em Dr. Peter Gehr, Institut für Anatomie, Leiter des Nationalen Forschungsprogrammes 64

(Chancen und Risiken von Nanomaterialien) SNF, Bern

Dr Sandor Györik, Viceprimario Medicina interna, Ospedale Regionale Bellinzona e Valli, Bellinzona

Dr Gaudenz Hafen, médecin associé, MER, responsable de l'unité de pneumologie pédiatrique CHUV, Lausanne

Prof. Dr. med. Jürg Hammer, Leitender Arzt, Chefarzt - Stellvertreter Pädiatrie, Intensivmedizin und Pneumologie,

Universitäts-Kinderspital beider Basel

Dr. med. Andrea L. Hartmann, Facharzt für Arbeitsmedizin, Winterthur

Dr. med. Erich Helfenstein, Pneumologie und Innere Medizin FMH, Luzern

Dr. med. Markus Hofmann, Allgemeinmedizin, Arbeitsmedizin FMH, Frauenfeld

Dr François Héritier, Président de la Société Suisse de Médecine Générale, Vice-président des Médecins de famille Suisse, Courfaivre JU

Dr. med. Peter Kälin, Allgemeine Medizin FMH, Sportmedizin SGSM, Manualmedizin SAMM,

Präsident der Ärztinnen und Ärzte für Umweltschutz, Leukerbad

Dr. med. Rainer M. Kaelin, Pneumologie und Innere Medizin FMH, Morges, Mitglied des Initiativkomitees

Dr Philippe Kehrer, Pneumologie FMH, Genève

Dr. med. Bruno Knöpfli, FMH Pädiatrie, Pneumologie und Sportmedizin, Davos

Prof. Dr. med, PhD. Nino Künzli, Vizedirektor Schweizerisches Tropen- und Publik Health Institut Universität Basel.

Mitglied Initiativkomitee 
Dr. med. Max Kuhn, Leitender Arzt Pneumologie Kantonsspital Graubünden, Chur

Dr. Med. RolfLindt, Lyss

Dr. med. Stefan Minder, Pneumologie FMH, Salemspital. Bern

PD Dr. med. Alexancer Möller, Leiter Pneumologie, Universitäts-Kinderkliniken Zürich

Dr. med. Marc Müller FMH Innere Medizin,

Vorstandsmitglied Schweizerische Gesellschaft für Allgemeinmedizin und Hausärzte Schweiz, Grindelwald

Prof. Dr Laurent Nicod, Médecin Chef Division de Pneumologie, CHUV, Lausanne

Dr Reto Olgiati Médecine interne et Pneumologie FMH, Délémont, JU

Dr. med. Helmut Oswald, FMH Kinder- und Jugendmedizin, spez. Lungenkrankheiten, Winterthur

Prof. Dr Arnaud Perrier, Médecin chef de service, Medecine interne Générale, Hôpital Universitaire, Genève

Dr. med. Max Pfenninger, Pneumologie FMH, Olten

Dr Franco Quadri, Medicina interna e malattie polmonari FMH. Capo Servizio di Pneumologie,

Ospedale Regionale Bellinzona e Valli, Bellinzona

Dr. med. Markus Riederer, FMH Pneumologie und Innere Medizin, Leitender Arzt Medizin RSE, Burgdorf, Präsident des Vereins der Pneumologen des Kantons Bern

Dr Philippe Rieder. FMH Médecine interne et Pneumologie, Médecin chef Hôpital de St.-Loup, VD

Prof. Dr Thierry Rochat, Médecin Chef, Service de Pneumologie. HUG. Genève

Dr Jean-Pierre Roulin, FMH Pneumologie, Fribourg

Dr. med. Jürg Rufener, FMH Innere Medizin, Vorstandsmitglied SGIM/SSMI, Interlaken

Prof. Dr. med. Ralph Alexander Schmid, Universitätsklinik für Thoraxchirurgie, Inselspital Bern

Prof. Dr. med. Martin Schöni, Chefarzt Ambulante Pädiatrie, Universitäts-Kinderklinik, Inselspital Bern

Dr Ariane Studer, Médecine interne générale FMH, Lausanne

Prof. Dr. Michael Tamm, Chefarzt Pneumologie, Universitätsspital, Basel

Dr Bernard Thiévent, Pneumologie FMH, Porrentruy

Prof. Dr. Med. Robert Thurnheer, Pneumologie FMH, Scherzingen

Dr Christophe Uldry, Médecin Chef, Service de Pneumologie et Réhabilitation Hôpital de Rolle

Dr. med. Silvia Ulrich Somaini,PD, FMH Pneumologie, Zürich

Dr. med. Beat Villiger, Pneumologie, FMH, Ärztlicher Leiter Medizinisches Zentrum Bad Ragaz

Dr Jacques Wacker, Medecine interne et Pneumologie FMH,La Chaux-de-Fonds

Dr Renato Waldenburger, Chefarzt Innere Medizin und Pneumologie FMH, Kantonales Spital Appenzell Ausserrhoden

Dr Jean Pierre Zellweger, FMH Pneumologie, Fribourg 


\section{Références}

1 www. who.int/tobacco/framework

2 Initiative parlementaire «Protection de la population et de l'économie de la fumée passive», Rapport de la commission de la sécurité sociale et de la santé du conseil national, $1^{\text {er }}$ juin 2007.

3 Gugger M, Cerny T, Barben J, Karrer W, Kaelin RM. Etre crédible!, Bull Méd Suisses. 2008;89(1/2): 22-3.

4 Communication aux média de la commission de la sécurité sociale et de la santé du Conseil des Etats, 9.1.2009 concernant la séance du 8.9.2008.

5 Les citations proviennent des procès verbaux des débats parlementaires, bulletin officiel www.admin. ch/parlement.

6 L'alliance est constitué de 53 organisations de santé, de sociétés médicales, de la FMH, la ligue pulmonaire Suisse, la ligue contre le cancer Suisse, etc. Voir www. rauchfrei-ja.ch/de/allianz-komitee/allianzpartner. html

7 Bonetti PO, Trachsel LD, Kuhn MU, Schulzki T, Erne P, Radovanovic D, Reinhart H. Incidence of actuel myocardial infarction after implementation of a public smoking ban in Graubünden, Switzerland. Two year follow-up. Swiss Med Wkly, 2011;141:w 13206. www. smw.ch

8 Pascal Couchepin, le 14.9.2008, Conseil des Etats: «... si au moins législation il y a, qu'elle soit simple et qu'elle ne provoque pas des disputes supplémentaires à propos du contrat de travail avec les collaborateurs et à propos des surfaces. La solution de la minorité est plus simple. Je m'excuse de vous avoir indigné, Monsieur Schwaller. J'en suis désolé, j'en ai honte, mais finalement, j'aurais honte de la législation, si elle passait telle que la majorité la propose.»

9 Didier Burkhalter, le 22.12.2011, Conseil national: «... le handicap le plus sérieux, c'est le manque de protection d'une partie des employés de la restauration avec le service dans les fumoirs. (...) Mais comme on l'a $\mathrm{vu}$, la loi actuelle permet de protéger une très grande partie de la population, tant sur les lieux de travail que dans les lieux fermées accessibles au public. L'initiative irait donc plus loin, de manière nationale...» Et: «Le conseil fédéral s'est battu en 2007 et 2008 pour une législation qui allait même plus loin que celle votée finalement par le parlement. Mais le parlement a décidé (...) (et), la mise en application a été faite il y a peu de temps. Elle se passe bien et les effets sont positifs.

10 Barben J, Borer H, Brändli O, Kaelin RM et nombreux co-signataires: Etre crédible II: la prévention en terrain miné. Au sujet des débats du 11.6.2008 au conseil national. Bull Méd Suisses. 2008;89:(38).1614-6.

11 Sur internet on trouve des agences qui proposent à des jeunes de sponsoriser leurs fêtes, sans frais pour nourriture, boissons et cigarettes.

12 L'industrie du tabac invite des jeunes adultes de participer aux études pour «tester leurs produits».

13 Le sponsoring de films et des productions télévisées par l'industrie du tabac est systématique. Des articles en Thorax,Vol 66. 10, Oct 2011 documentent l' efficacité de ces mesures: Waylen AE et al. Cross-sectional association between smoking depictions in films and adolescent tobacco use in a British cohort study; Hunt K et al. Exposure to smoking in films and own smoking among Scottish adolescents: a cross-sectional study. Morgenstern M et. Al. Smoking in movies and adolescent smoking: cross-cultural study in six European countries.

14 Roger Keller, Theda Radtke, Hans Krebs, Rainer Hornung: Der Tabakkonsum der Schweizer Wohnbevölkerung in den Jahren 2001-2010. Zusammenfassung des Forschungsberichtes 2011. Psychologisches Institut Universität Zürich.

15 Selon le vote éléctronique votèrent le rejet de l'initiative (Conseil national 22.12.2011)

Verts-lib.: 9 oui/0 non; abst.: 0

Chr.-dém.: 19 oui/ 6 non; abst.: 2

Bourg.dém.: 8 oui/0 non; abst.: 0

Vert: 3 oui/10non; abst.: 2

PLR: 25 oui/0 non; abst.: 1

PS: 2 oui/39 non; abst.: 4

SVP: 51 oui/0 non; abst.: 0

Total: 118 oui/55 non; abst.: 9

16 Kaelin RM, Diethelm P. Voting of members of Swiss Parliament during elaboration of federal smoking ban of Oct. 2008: which parties are crucial for decisions on public health measures? Poster 287, SGIM, Mai 2011. Swiss Medical Forum, suppl. 55, 2011;11(18).

17 Kaelin RM, Diethelm P. Efforts of Industry to influence Tobacco Control Policy in Switzerland. Poster SGIM Mai 2010.

18 Cette alliance (www.aepm.ch), dont le sécrétariat est assuré par le directeur de l'USAM Hans-Ulrich Bigler, est constituée par les organisations suivantes: USAM, Economiesuisse, ASCO (Association suisse des cafés-concerts, cabarets, dancings et discothèques), Gastrosuisse, Hotelleriesuisse, Union Suisse des paysans, Fédération Suisse des casinos, Publicité Suisse, Union patronale Suisse, Communauté du commerce suisse en tabacs, Association Suisse des fabricants de Cigares, VISCOM Association Suisse pour la communication visuelle), et d'autres Les organisations suivantes soutiennent l'AEPM: les partis politiques PDC et UDC, et Swiss Cigarette (qui regroupe Philip Morris internat., British American Tobacco internat et Japan Tobacco internat.)

19 Que les associations des restaurateurs se battent contre les interdictions de fumée est une constante du débat, depuis les années 80, en Californie (voir Fogel's Testimony, devant le conseil de la ville de New York.: «Il n'y avait pas d' association des restaurateurs avant l' interdiction de la fumée à Beverly Hills - nous avons été organisés par l' industrie du tabac.») La prise d'influence sur Gastrosuisse et sur HoReCa - international domicilié en Suisse, est prouvée par de documents de l' industrie du tabac de 1994 à 1995. Voir références on line à Lois sur le tabagisme passif: La Bavière s' est prononcée contre la confusion espagnole. Bull Méd Suisses. 2010;91(41):1618-20. 